\title{
Quantitative evaluation of patient-specific conforming hexahed ral meshes of abdominal aortic aneurysms and intraluminal thrombus generated from MRI
}

\author{
J. Tarjuelo-Gutierrez
}

\author{
B. Rodriguez-Vila
}

\author{
D.M. Pierce T. Fastl and E.J. Gomez
}

\begin{abstract}
A novel method for generating patient-specific high quality conforming hexahedral meshes is presented. The meshes are directly obtained from the segmentation of patient magnetic resonance (MR) images of abdominal aortic aneurysms (AAA). The MRI permits distinguishing between structures of interest in soft tissue. Being so, the contours of the lumen, the aortic wall and the intraluminal thrombus (ILT) are available and thus the meshes represent the actual anatomy of the patient's aneurysm, including the layered morphologies of these structures. Most AAAs are located in the lower part of the aorta and the upper section of the iliac arteries, where the inherent tortuosity of the anatomy and the presence of the ILT makes the generation of high-quality elements at the bifurcation is a challenging task. In this work we propose a novel approach for building quadrilateral meshes for each surface of the sectioned geometry, and generating conforming hexahedral meshes by combining the quadrilateral meshes. Conforming hexahedral meshes are created for the wall and the ILT. The resulting elements are evaluated on four patients' datasets using the Scaled Jacobian metric. Hexahedral meshes of 25,000 elements with $94.8 \%$ of elements well-suited for FE analysis are generated.
\end{abstract}

Keywords - magnetic resonance imaging, abdominal aortic aneurysm, intraluminal thrombus, conforming hexahedral meshes, finite element analysis.

\section{INTRODUCTION}

An aortic aneurysm is a localized dilation of the aorta that can be found anywhere in the artery, the most common being the abdominal aortic aneurysms (AAA) [1]. Weakening of the aortic wall is one of the biggest risks associated with this particular disease, which can lead to rupture or dissection of the artery. It is possible that blood stagnates in the dilation, inducing intraluminal thrombus (ILT) formation [2]. Three different structures may be observed in the aorta zone where an aneurysm is present: AAA wall, ILT and lumen.

Evaluating the rupture risk is a critical task due to the high mortality associated to this pathology [3]. Solutions based on the finite element method (FEM) and fluidstructure interaction (FSI) modeling can be used to evaluate rupture risk [4]. FE simulations for AAAs can be patientspecific, and permit modeling complex stress states that include the effect of the ILT. They also improve local environment representation based on preoperative anatomical images.

Generating an appropriate FE mesh is a pre-requisite for applying several numerical techniques, including those based on FEM [5]. Hexahedral meshes are preferred to tetrahedral ones due to: first, a model with the same volume is comprised of significantly less hexahedra than one made of tetrahedra; and second, there is an aspect ratio distortion present in tetrahedral solutions and not in hexahedral solutions [6], what makes hexahedra more suitable for applying FEM. However, in the specific case of vascular structures the use of hexahedral meshes is specially challenging due to the complex 3D branching topology. Hence, a specific algorithm is proposed to work with the geometry of the particular case study obtained from the patients' preoperative imaging. In our context, an aneurysm is characterized by its location and shape. About $90 \%$ of abdominal aneurysms are located below the renal arteries [1]. Furthermore, around two out of three abdominal aneurysms are located not only in the aorta, but extend to one or both iliac arteries [7]. Thereby, the geometry under study can be roughly described by a tubular structure in the shape of an inverted $\mathrm{Y}$, distorted by the aneurysm and ILT wherever they are present.

Most publications in literature which focus on this topic deal with planar bifurcations, i.e. centerlines evolving in a single plane [8-9] which do not represent the actual anatomy of the structures, but refined approximations. De Santis et al [10] propose a method for modeling structures evolving out of a single plane, where conforming meshes of the wall and the lumen are presented, but the outer contour of the wall cannot be segmented and is reconstructed from anatomical data.

In this study we present a novel and robust procedure for generating hexahedral conforming meshes of the aortic wall and the ILT with high-quality elements at the bifurcation, suitable for FEM analysis of stress states. The developed algorithm takes into account the evolution of the vessel's 
centerline out of a single plane and is constructed directly from segmented images, not needing reconstructed triangular surfaces.

\section{MATERIAL AND METHODS}

A technique for generating two conforming hexahedral meshes, one for the AAA wall and another one for the ILT, is presented. Starting from the manual segmentation of MR images, three quadrilateral meshes are constructed: one for the lumen, another for the aortic wall, and another one for the $\mathrm{ILT}$, wherever it is present. These quadrilateral meshes are combined in order to construct the final hexahedral meshes. Generating regularly shaped hexahedral elements at irregular anatomic structures such as bifurcations is a challenging task. In this work we present a novel method to handle the meshing at bifurcation producing high-quality elements. The algorithm has been implemented using MATLAB R2009a (Mathworks Inc., Natick, MA, USA) and the resulting meshes have been evaluated by applying the Scaled Jacobian metric.

\section{A. Image segmentation}

A novel MRI acquisition protocol which provides high contrast between the aortic wall and the ILT is used. This protocol makes it possible to distinguish and segment the different structures of interest. Hence, three 3D binary images of the wall, the ILT and the lumen are obtained for each patient using the ITK-SNAP software [11].

\section{B. Quadrilateral meshes generation}

The initial phase for modeling the bifurcation consists of dividing it into three different vessels, following the idea proposed by Lee [12]. Once the vessels have been separated, each one is divided into two sections that are modeled independently, so the quality and number of elements can be tuned at each section, depending on the anatomical characteristics of each section. By doing so, the computational cost of the process is minimized as the density of elements is increased only where necessary. The tuning is controlled by a set of parameters that divide the sections in the longitudinal and circumferential directions.

Three quadrilateral meshes are constructed for the external face of the aortic wall (Fig. 1a), internal face of the aortic wall (Fig. 1b) and the lumen (Fig. 1c), after determining the sections and setting the parameters. The process for generating each mesh is detailed next. The initial axial contours are divided according to the circumferential parameters, which establish points equally spaced over the contours. The union of the corresponding points from each axial contour results in a set of longitudinal lines. Then these lines are divided by applying the longitudinal parameters. In this case, the resulting points are not equally spaced along the longitudinal lines due to the dilation of the bifurcation. They are placed further apart the closer they are to the bifurcation. Again, the union of corresponding points results in new set of circumferential lines. The combination of longitudinal and circumferential lines provides the quadrilateral mesh. In order to build hexahedral meshes from the quadrilateral ones, a set of center points must be available. These points are established as midpoints of the extreme longitudinal lines.
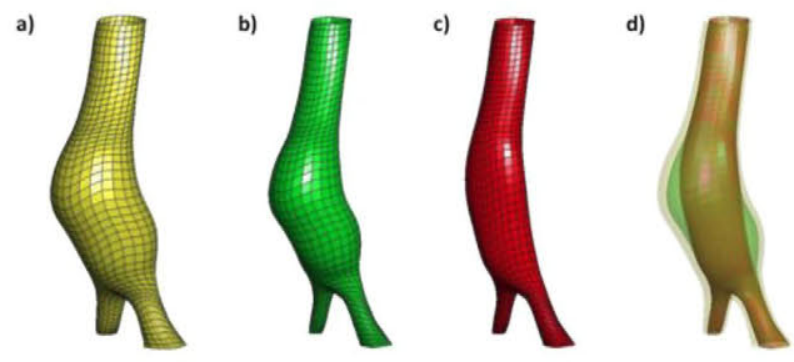

Fig. 1 Quadrilaterals meshes of a) external face of the wall b) internal face of the wall c) lumen. d) Hexahedral meshes of the wall and the ILT

\section{Hexahedral meshes generation}

The necessary elements for the construction of hexahedral meshes are the central points and the three quadrilateral meshes; one of them is taken as reference. The choice of the reference mesh is arbitrary: in this case we have chosen the wall's external face mesh. In order to avoid the formation of distorted hexahedra, the quadrilaterals of the remaining meshes must be refined. This improvement, performed using the ray casting technique, is based on the reference mesh. The hexahedral mesh of the wall is built by joining the quadrilaterals of the external and internal faces of the wall. The process is repeated likewise for the generation of the hexahedral mesh of the ILT, taking as reference in this case the just refined quadrilateral mesh. Any hexahedra with no volume (wherever the ILT does not exist, so the internal wall's face is equal to the lumen) are removed (Fig. 1d).

\section{Evaluation of mesh quality}

Within the context of the FE method, the quality of the finite elements obtained from the mesh generation greatly affects both the convergence of the simulations and the resulting approximations to the solutions of the governing 
partial differential equations. Additionally, accuracy in the representation of the true, patient-specific in vivo geometry also influences the applicability of the results [13]. Metrics for mesh quality must detect inverted elements (elements which generate meaningless results) and provide an estimate of the mesh's fitness for use in numerical simulations.

For the analysis for solid structures the Scaled Jacobian is a common quality metric [10]. Scaled Jacobian takes the range $[-1,1]$ for a hexahedral element, -1 corresponding to the worst possible elements and +1 the best possible ones. The Scaled Jacobian applies only to regular hexahedral elements having eight (distinct) vertices at different spatial locations in 3D space. We use the open source program Paraview (Kitware, Inc., Clifton Park, New York, USA) to evaluate the Scaled Jacobian, among other quality metrics.

\section{REsUlts}

The performance of the technique described in the methodology was tested over a set of four MRI studies of patients presenting AAAs near the bifurcation of the iliac arteries. We are able to generate conforming hexahedral meshes, e.g. containing 25,000 elements in approximately $30 \mathrm{sec}$ using a PC with $3 \mathrm{GHz}$ Core Duo Pentium, $8 \mathrm{~GB}$ RAM and a 64 bit OS.

Fig. 2 illustrates in a qualitative way the resulting conforming hexahedral meshes of the arterial wall, while Fig. 3. illustrates their corresponding ILTs (not to scale). The meshes in Figs. 2 and 3 are shaded according to the Scaled Jacobian of the mesh elements.
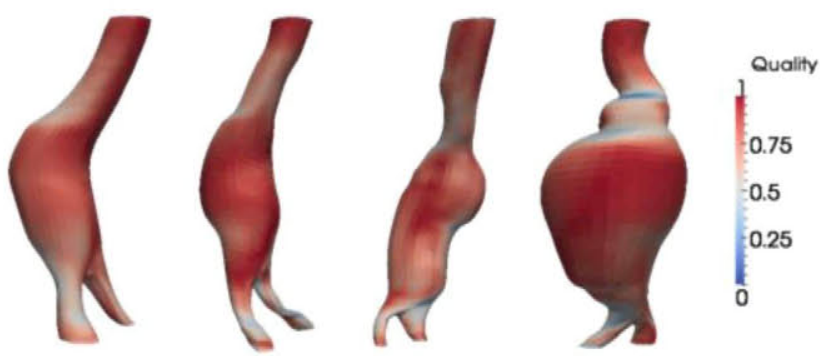

Fig. 2 Results for four representative Abdominal Aortic Aneurysms (AAAs) walls using the Scaled Jacobian as a measure of element quality.

Table 1 presents a quantitative description of the element's quality distribution. The defined ranges for this distribution represent from the unacceptable results $(<0.0)$ until the excellent quality elements $(0.8-1.0)$. Both the distribution of the Scaled Jacobian values for the separated layers of the AAA walls (intima, media and adventitita), and the separated layers of the ILTs (luminal, medial and abluminal) are illustrated for the four representative MRI studies. The representation is in terms of the arithmetic means and the standard deviations. Elements with Scaled Jacobian values in the range of 0.5 to 1.0 are well-suited to FE analysis [9].
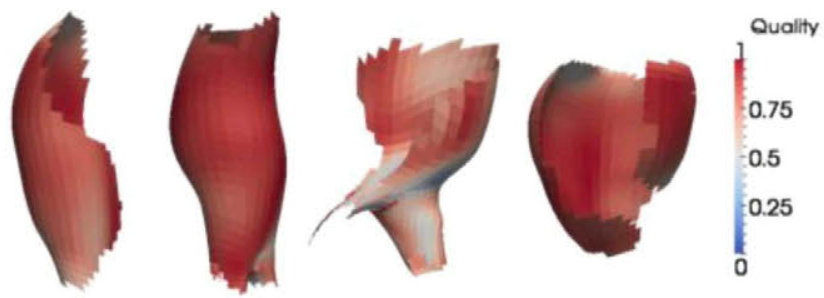

Fig. 3 Results for four representative Abdominal Aortic Aneurysms (AAAs) intraluminal thrombi (ILTs) using the Scaled Jacobian as a measure of element quality.

Table 1 Distribution frequencies (\%) of scaled Jacobian values for individual layers in AAA wall and AAA thrombus, expressed as the arithmetic mean (AM) and the standard deviation (SD) of the patient set

\begin{tabular}{l|l:l|l|l|l|l|l|l|l|l|l|l|l|l|}
\multirow{2}{*}{ Scaled Jacobian } & \multicolumn{2}{|c|}{$<$} & \multicolumn{2}{|c|}{$0.0-2$} & \multicolumn{2}{|c|}{$0.2-0-4$} & \multicolumn{2}{|c|}{$0.4-0.6$} & \multicolumn{2}{|c|}{$0.6-0.8$} & \multicolumn{2}{|c|}{$0.8-1.0$} \\
& $\mathrm{AM}$ & $\mathrm{SD}$ & $\mathrm{AM}$ & $\mathrm{SD}$ & $\mathrm{AM}$ & $\mathrm{SD}$ & $\mathrm{AM}$ & $\mathrm{SD}$ & $\mathrm{AM}$ & $\mathrm{SD}$ & $\mathrm{AM}$ & $\mathrm{SD}$ \\
\hline Adventitia & - & - & 2.05 & 3.42 & 7.86 & 5.70 & 18.65 & 3.27 & 36.36 & 2.53 & 35.08 & 7.21 \\
Media & - & - & 1.52 & 3.24 & 6.97 & 6.05 & 19.18 & 3.66 & 37.17 & 1.96 & 35.16 & 7.81 \\
Intima & - & - & 1.29 & 2.80 & 6.34 & 6.22 & 19.74 & 4.06 & 37.81 & 2.84 & 34.82 & 7.95 \\
\hline Abluminal & - & - & 0.83 & 1.85 & 3.49 & 4.33 & 16.97 & 11.41 & 35.42 & 12.21 & 43.29 & 25.81 \\
Medial & - & - & 1.05 & 2.35 & 2.01 & 3.13 & 13.16 & 11.07 & 34.44 & 15.19 & 49.35 & 28.75 \\
Luminal & - & - & 1.43 & 2.44 & 2.05 & 2.22 & 11.21 & 11.90 & 37.39 & 14.85 & 53.92 & 29.46 \\
\hline
\end{tabular}




\section{Discussion}

Negative values signify invalid (inverted) elements illsuited to FE analysis. For the ILTs, the element quality is generally at a very high level: there are no elements with negative Scaled Jacobian values and $94.8 \%$ of the total elements exhibit Scaled Jacobian values greater than 0.5. Thrombi of different shapes and located at different heights of the artery are represented. Importantly, note that the (layered) wall shapes are generally more complex than the thrombi shapes. Nonetheless, the elements of the AAA wall are also of high quality: $87.8 \%$ of elements in the wall exhibit Scaled Jacobian values greater than 0.5; meanwhile only $0.22 \%$ fall below 0.2 . In the examples shown, we use approximately 25,000 elements per mesh (suitable for FE analysis in less than one week).

The relatively low quality elements do not occur in the region of the bifurcation, but rather at regions where the surfaces of the original geometry lie relatively parallel to the original MR Imaging planes, due either to the presence of the ILT or to the tortuosity inherent to the arteries, as illustrated in Fig. 4.

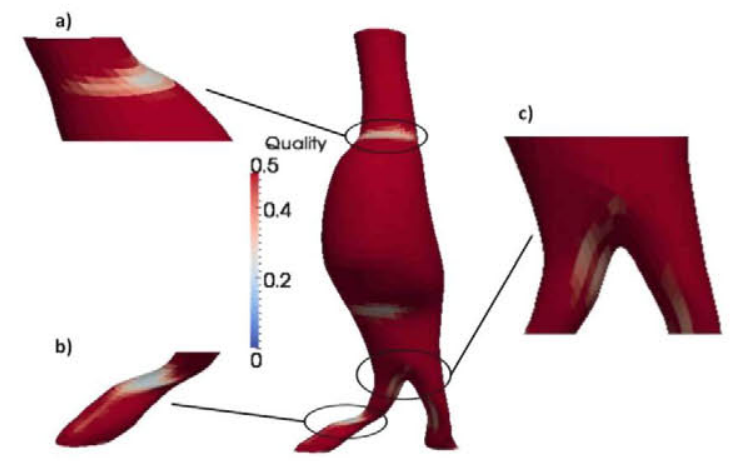

Fig. 4 Representative result for a patient-specific AAA: Scaled Jacobian shown with range $0.0-0.5$. Relatively low quality elements do not occur in the region of the bifurcation (c), but rather at regions where the surfaces of the geometry lie relatively parallel to the original MRI planes(a-b).

\section{v. CONClusions}

In this work we propose a novel procedure for generating patient-specific conforming hexahedral meshes of AAAs and their corresponding thrombi with high-quality elements, particularly in the area of the bifurcation into the iliac arteries. These structures are divided into their layered morphologies. By doing so we provide novel input for simulations, as the layers have different histological and mechanical properties, representing the actual anatomy of the structures.

\section{ACKNOWLEDGMENT}

The authors gratefully acknowledge the financial support of the EC under the 7th Framework Programme in the frame of project SCATh, FP7-ICT-2009-4-248782.

\section{REFERENCES}

1. Fauci AS, Braunwald E, Kasper DL, Hauser SL, Longo DL, Jameson JL, Loscalzo J (2012) Harrison's Manual of Medicine. 18th ed. McGrawHil, New York, NY

2. Gloviczki P, Ricotta JJII (2007) Aneurysmal vascular disease. In: Townsend CM, Beauchamp RD, Evers BM, Mattox KL (eds) Sabiston Textbook of Surgery. 18th ed., Philadelphia, PA, Saunders Elsevier: chap 65

3. European cardiovascular disease statistics (2012). published by the European Heart Network

4. Venkatasubramaniam AK, Fagan MJ, Mehta T, Mylankal KJ, Ray B, Kuhan G, Chetter IC, McCollum PT (2004) A comparative study of aortic wall stress using finite element analysis for ruptured and nonruptured abdominal aortic aneurysms. Eur J Vasc Endovasc Surg 28(2): $168-176$

5. Ho-Le K (1988) Finite element mesh generation methods: a review and classification. Comput Aided Design 20(1):27-38

6. Ramos A, Simões JA (2006) Tetrahedral versus hexahedral finite elements in numerical modeling of the proximal femur. Med Eng Phys. 28(9):916-924

7. Wassef M, Baxter BT, Chisholm RL, Dalman RL, Fillinger MF, Heinecke J (2001) Pathogenesis of abdominal aortic aneurysms: a multidisciplinary research program supported by the National Heart, Lung, and Blood Institute. J Vasc Surg 34(4):730-738

8. Antiga L, Ene-Iordache B, Caverni L, Cornalba GP, Remuzzi A (2002) Geometric reconstruction for computational mesh generation of arterial bifurcations from CT angiography. Comput Med Imaging Graph 26(4):227-235

9. Antiga L, Steinman D (2004) Robust and objective decomposition and mapping of bifurcating vessels. IEEE Trans Med Imag 23(6):704-713

10. De Santis G, De Beule M, Segers P, Verdonck P, Verhegghe B (2011) Patient-specific computational haemodynamics: generation of structured and conformal hexahedral meshes from triangulated surfaces of vascular bifurcations. Comput Methods Biomech Biomed Engin 14(9):797-802

11. Yushkevich PA, Piven J, Hazlett HC, Smith RG, Ho S, Gee JC, Gerig $\mathrm{G}$ (2006) User-guided 3D active contour segmentation of anatomical structures: Significantly improved efficiency and reliability Neuroimage 1(3): 1116-28.

12. Lee SE, Piersol N, Loth F, Fischer P, Leaf G, Smith B, Yedevalli R, Yardimci A, Alperin N, Schwartz L (2000) Automated Mesh Generation of an Arterial Bifurcation Base upon in vivo MR Images. In: Engineering in Medicine and Biology Society. Proceedings of the 22nd Annual International Conference of the IEEE, Chicago, IL, Vol.1, pp 719-722

13. Knupp P (2007) Remarks on mesh quality. In: Proceedings of AIAA 45th Aerospace Sciences Meeting and Exhibit, Reno, NV

Author: Jaime Tarjuelo Gutiérrez

Institute: Bioengineering and Telemedicine Centre, ETSI de Telecomunicación, Universidad Politécnica de Madrid

Street: Avenida Complutense 30

City: Madrid

Country: Spain

Email: jtarjuelo@gbt.tfo.upm.es 